\title{
$1 /$ Parmi les ressources mobilisées par l'étudiant pour planifier son stage, quel rôle réserve-t-il au formateur institutionnel?
}

\author{
Agnès Deprit, Virginie März et Catherine Van Nieuwenhoven \\ Université catholique de Louvain
}

L'articulation théorie-pratique au moment de la préparation d'un stage est difficile pour l'étudiant tant il perçoit un écart entre les savoirs appris et la réalité du terrain (Caron \& Portelance, 2017). Afin d'observer si le formateur est la ressource par laquelle l'étudiant réalise ces liens, une recherche exploratoire a permis, par le biais d'un questionnaire écrit, d'identifier les ressources quil mobilise prioritairement pour préparer son stage. L'analyse montre que l'étudiant se détache progressivement du formateur et de ses cours pour utiliser, en fin de cursus, des ressources davantage externes à la formation (bibliothèque et Internet).

\section{Problématique}

La formation des instituteurs primaires en Belgique francophone est définie légalement (Décret 2000) et prévoit l'organisation de stages pour permettre au futur enseignant d'acquérir le savoir-faire professionnel qui repose sur l'articulation théorie-pratique. Ces moments d'articulation vont au-delà d'une simple application en contexte réel des savoirs appris. Il s'agit, pour Correa Molina, Gervais et Rittershaussen (2008), de véritables espaces d'intégration et de développement de compétences professionnelles où le potentiel de l'apprenant (théories acquises, expériences antérieures, habiletés personnelles, etc.) se révèle dans l'action (Gervais, 2008).

Or, l'articulation théorie-pratique semble poser problème à ce moment précis où les enseignants des instituts de formation et les maitres de stage attendent un réinvestissement des savoirs enseignés. En effet, alors que les formateurs 1 encouragent implicitement l'autonomie et la responsabilisation du stagiaire dans l'établissement de ces liens, les étudiants vivent des difficultés et sont en attente d'aide (Caron \& Portelance, 2017) tant l'écart entre les théories apprises et la réalité du terrain leur parait discordant (Caron \& Portelance, 2017; Clerc \& Martin, 2011; Waege \& Haugaløkken, 2013). 
Peu d'études se sont penchées sur la manière dont l'étudiant gère cette phase préactive, définie par Clerc et Martin (2011) comme le moment de la planification de l'intervention, ni sur la façon dont les compétences professionnelles s'y construisent. Ce constat est notamment relayé par Dessus (2002) qui mentionne un nombre restreint de recherches empiriques relatives à l'importance de la planification pour l'activité enseignante. Par conséquent, comprendre la manière dont l'étudiant se prépare pour ses stages, le considérer selon ses atouts et son expertise (Fleming, 2014; Ulvik \& Langørgen, 2012) constitue une pertinence pour la recherche scientifique et un enjeu important dans le contexte actuel de la refonte de la formation initiale des enseignants en Belgique francophone (Académie de recherche et d'enseignement supérieur [ARES], 2017) afin d'adapter au mieux le nouveau curriculum de formation aux caractéristiques des apprenants.

Récemment, Deprit et Van Nieuwenhoven (2018) ont identifié dix ressources que les futurs instituteurs primaires en dernière année de formation utilisent pour préparer leur stage. Elles ont constaté que les ressources délaissées (les formateurs et les cours) semblent révéler que les savoirs théoriques sont faiblement mobilisés. Gervais et Leroux (2011) observent également un désintérêt des savoirs académiques chez les stagiaires de l'enseignement secondaire bien qu'ils soient davantage sollicités au moment de la conception des situations d'apprentissage et d'enseignement. Ces nuances pourraient provenir d'un rapport différent au savoir entre ces deux niveaux d'enseignement.

À ces constats, Piot (2008) ajoute que les savoirs théoriques sont faiblement opérationnels chez l'enseignant novice, ce qui laisse supposer qu'ils le sont peu également chez les stagiaires. C'est l'accumulation d'expériences réflexives menées sur l'action au moment où elle se déroule et en dehors du temps de classe (pré ou post-activement) qui les rend progressivement fonctionnels pour analyser une situation et la problématiser.

Cette problématique du réinvestissement des théories apprises au moment de la pratique est le cœur de la recherche exploratoire que nous avons menée auprès de futurs instituteurs primaires. Elle a pour but d'identifier, à travers les ressources utilisées par les étudiants lors de la préparation de stage, si les savoirs appris sont sollicités soit par des rencontres avec les formateurs, soit par la consultation de leurs notes de cours.

Après une approche théorique de l'étudiant évoluant dans son cursus de formation, la méthodologie qualitative choisie est présentée. Les résultats permettent d'observer si les savoirs appris sont réinvestis et si c'est par le biais de la ressource formateur qu'ils sont utilisés au moment de la planification. 


\section{Cadre conceptuel}

Pour accéder au point de vue de l'étudiant à travers les choix qu'il pose, il est nécessaire de comprendre qui il est. Deux éléments influençant sa perception et ses décisions sont d'abord présentés: son statut d'étudiant qui l'amène à poser des choix stratégiques; les ressources dont il dispose et qu'il décide, ou non, de mobiliser pour préparer ses activités. Ensuite, un focus est apporté à la ressource «formateur», accompagnateur privilégié de l'étudiant au moment de sa pratique et représentant des théories enseignées, afin d'approcher au mieux la problématique.

\section{L'étudiant pose des choix stratégiques}

Le parcours de l'étudiant en formation n'est pas linéaire. Selon une récente étude réalisée en Belgique francophone (Bernal Gonzalez et al., 2018), le futur enseignant traverserait trois phases de développement professionnel durant sa formation initiale. La première correspond à l'entrée dans le cursus. À ce moment, l'étudiant aurait une vision peu développée du métier pour lequel il est venu se former, ne percevant les tâches de l'enseignant que dans leur globalité. La phase suivante est observée au cours de la deuxième année de formation. Suite à une pratique qui s'intensifie, il vivrait une période d'insécurité, de résistance envers la formation, se sentant principalement démuni pour assurer la gestion de la classe. Par la suite, progressivement mieux outillé, une phase d'espoir est observée et serait caractérisée par la prise en main du stagiaire qui aurait alors une vision plus précise de son futur métier et vivrait une sorte de sérénité face à sa capacité à enseigner.

Cette compréhension graduelle du métier pourrait avoir un impact sur les décisions qu'il prend, notamment sur le choix des ressources qu'il considère utiles pour préparer son stage et qui le mèneront, d'après lui, à la réussite (Dweck, 2003). Ainsi, il use de diverses stratégies. Le jeune reproduit les techniques de travail qu'il a utilisées dans son parcours scolaire antérieur et qui ont porté leurs fruits. C'est le cas du bachotage ou du travail solitaire et autocentré soulignés dans de récentes enquêtes et études françaises (Beaupère, Boudesseul, \& Macaire, 2009; Paquelin, 2016). L'étudiant aime travailler seul et coopère peu, les véritables relations de travail ne s'observant que chez les étudiants les plus studieux (Monfort, 2006). Néanmoins, il cherche de l'aide auprès de ses pairs s'il se retrouve en difficulté et ne sollicite ses enseignants qu'en dernier recours (Paquelin, 2016).

Ses choix, portés sur les ressources accessibles, sont guidés par trois principes (Boubée, 2011, octobre): le principe du moindre effort physique ou cognitif, le principe de suffisance (une information est satisfaisante car jugée suffisante) et le principe d'utilité qui privilégie, selon Savolainen (1999), les stratégies de recherche antérieures qui ont fait leur preuve. 
L'étudiant est également dirigé par l'urgence des situations qui le mène à préférer, en contexte de stage, les savoirs opératoires aux savoirs savants (Martin, 2004). À ce moment, l'offre proposée par le maitre de stage rencontre les attentes du stagiaire si bien que leurs discussions se situent au niveau pratique en se basant sur le bon sens et les règles personnelles plutôt que sur des savoirs reconnus comme dérivés de la recherche qu'il faudra encore, selon Perrenoud (2004), transposer pragmatiquement pour les rendre accessibles aux élèves.

Pour cette recherche, nous retenons que l'étudiant est libre de ses choix et stratège dans les décisions raisonnées qu'il prend en fonction du contexte matériel, structurel et humain dans lequel il évolue. Ainsi, nous approchons l'étudiant selon la perspective non déficitaire de Malo $(2005,2010)$ qui consiste à adopter une double position. Il s'agit, d'une part, de considérer le point de vue du stagiaire, d'accepter sa compréhension de la situation puisque sa marnière de lire les évènements a du sens pour lui. D'autre part, il convient de le regarder différemment, de manière positive - d'apprécier ses atouts, ses talents (Colognesi, Beausaert, \& Van Nieuwenhoven, 2018) - pour comprendre qu'à travers ses décisions il cherche à s'adapter à la situation, à trouver un équilibre viable entre les coûts et les bénéfices qu'il perçoit des interventions qu'il met en place pour résoudre un problème.

\section{L'étudiant possède un équipement de ressources pour agir}

Dans la littérature, les ressources que les enseignants utilisent pour agir ont fait l'objet de plusieurs structurations (Malo, 2005; Schön, 1983; Vause, 2011). Pour notre recherche, nous avons retenu la typologie du double équipement de Le Boterf (2003) en ce sens qu'elle permet non seulement, comme pour les typologies des auteurs précités, de saisir les ressources incorporées du futur enseignant mais aussi d'identifier tout un ensemble de ressources de l'environnement auxquelles il a accès.

Selon Le Boterf (2003), les ressources incorporées (première partie de l'équipement) sont constituées des savoirs théoriques - dont les valeurs et croyances, les savoirs procéduraux et sociaux -, du savoir-faire ainsi que de l'ensemble des caractéristiques personnelles de l'individu telles que ses qualités, ses aptitudes ou ses ressources émotionnelles (Gervais \& Leroux, 2011). Notons que face à cet ensemble de savoirs incorporés, tout praticien va prioritairement activer ceux qui sont issus de l'expérience lorsqu'il élabore de nouvelles activités d'apprentissage (Schön, 1983; Vause, 2011).

Les ressources de l'environnement, ainsi nommées par Le Boterf (2003), constituent la seconde partie de l'équipement de l'enseignant. Gervais et Leroux (2011) parlent de ressources externes et les structurent en cinq branches: les réseaux relationnels, documentaires, informationnels et d'expertise ainsi que les ressources matérielles disponibles. Cette quantité considérable d'informations entraine une difficulté de traitement. Pour Le Boterf (2003), les praticiens sont 
peu outillés pour les gérer efficacement afin de les utiliser dans des activités ou dans la résolution des problèmes professionnels qui se présentent.

La compétence de planification qui nous intéresse ici ne se résume donc pas à disposer d'une série d'attributs, mais correspond à la faculté de gérer les ressources disponibles (incorporées et externes) et de les combiner de manière efficiente pour «savoir agir en situation» (Le Boterf, 2003).

En conséquence, il nous semble nécessaire de considérer l'étudiant selon ses capacités à choisir des ressources utiles pour planifier son stage. La décision de réinvestir des savoirs appris pour construire sa pratique fait partie de ses choix stratégiques. C'est la raison pour laquelle, dans les lignes qui suivent, nous zoomons sur la ressource formateur, garant théorique qui peut être sollicité en tant que personne ou à travers ses cours.

\section{Le formateur au moment de La préparation de stage, une ressource pour L'étudiant}

En se référant au double équipement de Le Boterf (2003), la ressource formateur est une ressource externe issue du réseau d'expertise. Elle est activable à la demande de l'étudiant, encouragé à se responsabiliser et à travailler de manière autonome quand il prépare son stage. Or, l'écart qu'il perçoit entre les savoirs appris et le terrain l'instabilise (Waege \& Haugaløkken, 2013), si bien que le formateur est amené à endosser le rôle de médiateur entre la théorie et la pratique pour lui venir en aide (Caron \& Portelance, 2017). Le formateur peut dès lors être considéré comme un secours pour l'étudiant qui planifie son stage, son rôle n'étant alors plus seulement de former mais d'accompagner.

Former de futurs enseignants c'est «inculquer [...] les connaissances, les compétences et les comportements dont ils ont besoin pour enseigner au niveau d'enseignement considéré» (Ria, 2016, p. 253). C'est donc les préparer à entrer dans un métier qui, aujourd'hui, est mouvant et difficile à définir clairement (Saujat, Saussez, \& Sève, 2016). De Ketele (2018, p. 17) parle d'un «travail de construction de la professionnalité» qui s'opère à la fois dans les instituts de formation et sur le terrain, auquel se joint un accompagnement réflexif pendant et sur l'action. L'accompagnement fait donc partie du processus de formation

Accompagner est «un art, pas une science: on l'apprend par la pratique, par ajustements successifs» (Vial \& Caparros-Mencacci, 2007, p. 36). Il s'agit en effet de créer une relation bienveillante et constructive qui s'inscrit dans le projet formel (Paul, 2016) ou informel (Colognesi, Beausaert, \& Van Nieuwenhoven, 2018) de l'accompagné. L'accompagnement nécessite donc d'entrer dans la perception de celui qu'on accompagne: comprendre son objectif, percevoir ce qui l'instabilise (Malo, 2010) et déceler ses talents (Colognesi et al., 2018) pour se mettre en route avec lui, partager son cheminement (De Ketele, 2018; Paul, 2016) vers ce qui rendra sa situation viable et fera, pour lui, équilibre (Malo, 2010). Accompagner ce n'est pas donc pas rechercher un rendement, un apprentissage (Paul, 2016), c'est avant tout établir une relation. 
Or, plusieurs auteurs soulignent combien le formateur, expert d'une discipline d'enseignement, n'est pas formé pour ce suivi. Ils précisent d'ailleurs qu'il s'agit pour lui d'une nouvelle fonction à endosser, voire d'un nouveau métier (Paquay, 2012; Van Nieuwenhoven, Picron, \& Colognesi, 2016). Aussi, en ajustant la posture à la situation d'interaction, le formateur favorise la réflexivité du futur professionnel, son autonomie et son émancipation.

Parmi les différentes typologies de postures existantes, celle de Mieusset (2013) se rapproche davantage de l'accompagnement du formateur car elle définit les postures du maitre de stage, autre partenaire privilégié de l'étudiant au moment de sa pratique et qui, de surcroit, fait aussi partie du réseau d'expertise, à savoir:

- apporter un conseil consiste à suggérer des idées, des outils ou à proposer des solutions pour aider le stagiaire dans les différentes tâches du métier;

- imposer une décision ne laisse pas le choix au stagiaire qui doit appliquer les idées, les outils et les points de vue de l'accompagnateur sur les tâches du métier;

- se positionner en second est la position du suiveur qui valorise l'accompagné, le laisse essayer, se met à son service pour favoriser son autonomie;

- soutenir, réconforter, rassurer assure une présence humaine auprès de l'étudiant fragilisé ou en difficulté.

Si les postures du maitre de stage centrent l'accompagnement sur la mise en œuvre du métier (idées, outils, tâches), elles ne portent pas sur l'articulation théorie-pratique. Ce rôle serait-il réservé au formateur? Devant ce questionnement, nous postulons que les postures du formateur apportent une coloration à l'accompagnement notamment dans l'aide au réinvestissement des théories apprises et que, de son côté, le stagiaire est en attente d'une posture spécifique en fonction des déséquilibres qu’il vit.

\section{Méthodologie}

Une recherche exploratoire a été menée pour répondre aux deux questions qui sous-tendent cette étude: que recherchent les étudiants dans les ressources qu'ils utilisent prioritairement au moment de préparer leur stage? Quelles sont les attentes de l'étudiant lorsqu'il mobilise la ressource formateur (personne-ressource et cours)?

La démarche choisie est mixte à visées descriptive et interprétative (Van der Maren, 1996; Savoie-Zajc, 2011) afin de comprendre les propos et la perception des étudiants selon l'approche non déficitaire adoptée (Malo, 2005, 2010). Le but est, rappelons-le, d'identifier l'utilisation des ressources au moment de la préparation de stage et plus spécifiquement la ressource liée au formateur (personneressource et cours) afin de déterminer en quoi il serait identifié, par l'étudiant, comme un facilitateur d'intégration de la théorie au moment du stage. 
L'échantillon est constitué de l'ensemble des étudiants, futurs instituteurs primaires, d'une implantation de la Haute École Louvain en Hainaut (HELHa, Belgique francophone) amenés, par décret (Décret 2000), à prendre en charge la classe de manière progressive au cours des deux semaines de stage qu'ils réalisent en $\mathrm{B}_{1}^{2}$, et de manière autonome en $\mathrm{B} 2$ et en $\mathrm{B} 3$ ( 4 et 10 semaines de stage). L'échantillon se répartit sur les trois années de formation et totalise 176 répondants (Tableau 1).

\section{Tableau 1: Répartition de l'échantillon sur les trois années de formation}

\begin{tabular}{c|c|c|c|c} 
HELHa - Bacheliers Instituteurs primaires & B1 & B2 & B3 & TOTAL \\
\hline Questionnaire AVANT le Stage 1 & 40 & 29 & 27 & 96 \\
\hline Questionnaire APRÈS le Stage 1 & 31 & 24 & 25 & 80 \\
\hline TOTAL & 71 & 53 & 52 & 176
\end{tabular}

L'outil de recueil est constitué de deux questionnaires auxquels les étudiants ont été invités à répondre, par écrit, à deux moments déterminés: le premier pendant la préparation du premier stage de l'année, le second durant les quelques jours qui ont suivi son terme. L'analyse de ce second questionnaire fera l'objet d'une publication ultérieure.

Le questionnaire propose aux étudiants de répondre à deux questions. La première: "Quelles sont les ressources que tu as utilisées pour préparer ton stage?» convie les étudiants à cocher, parmi les dix ressources proposées (Deprit \& Van Nieuwenhoven, 2018), celles qu'ils priorisent en hiérarchisant leur choix (question à choix multiples). La seconde les sollicite à expliquer ce choix (question ouverte).

L'analyse, effectuée sur la base des trois choix prioritaires de chaque étudiant, a été réalisée à deux niveaux. Le premier niveau a permis de classer, au sein de catégories émergentes, les objets que les étudiants recherchent dans les ressources exploitées pour préparer leur stage. Le second niveau d'analyse a permis de répartir les postures des formateurs que les étudiants sollicitent dans des catégories préétablies: les quatre postures de Mieusset (2013). Pour ce dernier classement, deux catégories émergentes ont été ajoutées ("prendre en main» et "collaborer») pour les verbatims ne s'insérant pas dans la typologie de départ, ce qui totalise six postures.

Afin de s'assurer de l'indépendance des codages par rapport aux chercheures, un accord inter-juges a été mesuré au moyen du Kappa de Cohen. Il souligne un accord fort ( $k=.772$ pour le codage des postures) à presque parfait $(k=.821$ pour le classement des objets de recherche), qui témoigne d'une fidélité intercodeurs satisfaisante. 


\section{Analyse des résultats}

Dans cette section, nous abordons l'analyse des résultats afin de mettre à jour ce que cherchent les étudiants quand ils utilisent des ressources pour préparer leur stage et ensuite, plus finement, leurs attentes lorsqu'ils utilisent la ressource formateur.

\section{Que recherchent les étudiants dans les ressources qu'ils utilisent prioritairement au moment de la préparation de leur stage?}

Pour répondre à cette question, nous avons identifié les ressources que les étudiants utilisent prioritairement pour préparer leur stage et ce dans les trois années de formation afin d'observer ce qu'ils recherchent dans leurs trois choix privilégiés.

\section{Les ressources privilégiées par les étudiants pour préparer leur stage}

Les résultats obtenus (Tableau 2) sont présentés en trois tableaux qui correspondent chacun à une année du cursus de formation et reprennent le classement hiérarchisé des ressources, de la plus souvent citée à la moins choisie.

Tableau 2: Classement hiérarchique des ressources évoquées au moment de la préparation de stage, pour les trois années de formation

\begin{tabular}{|c|c|c|c|c|c|}
\hline \multicolumn{2}{|l|}{$\begin{array}{l}\text { B1 PRIMAIRE } \\
46 \text { étudiants }\end{array}$} & \multicolumn{2}{|l|}{$\begin{array}{l}\text { B2 PRIMAIRE } \\
29 \text { étudiants }\end{array}$} & \multicolumn{2}{|l|}{$\begin{array}{l}\text { B3 PRIMAIRE } \\
27 \text { étudiants }\end{array}$} \\
\hline TOP & $\begin{array}{l}\text { Nbr } \\
\text { Etds }\end{array}$ & TOP & $\begin{array}{l}\text { Nbre } \\
\text { Etds }\end{array}$ & TOP & $\begin{array}{l}\text { Nbre } \\
\text { Etds }\end{array}$ \\
\hline 1 Cours HE & 29 & 1 Bibliothèque & 24 & 1 Bibliothèque & 20 \\
\hline 2 Formateurs & 25 & 2 Cours HE & 13 & 2 Internet & 15 \\
\hline 3 Bibliothèque & 21 & 3 Maître de stag & 13 & 3 Maître de stage & 10 \\
\hline 4 Maître de stage & 18 & 4 Formateurs & 12 & 4 Cours HE & 10 \\
\hline 5 Internet & 16 & 5 Mémoire & 11 & 5 Mémoire & 9 \\
\hline 6 Mémoire & -14 & 6 Internet & 7 & 6 Étudiants & 7 \\
\hline 7 Étudiants & 6 & 7 Anciennes leçons & 3 & 7 Anciennes leçons & 3 \\
\hline 8 Connaissances & 4 & 8 Cahiers Es & 2 & 8 Formateurs & 2 \\
\hline 9 Anciennes leçons & 3 & 9 Connaissances & 1 & 9 Cahiers Es & 2 \\
\hline 10 Cahiers Es & 1 & 10 Étudiants & 1 & 10 Connaissances & 1 \\
\hline
\end{tabular}

Légende: plusieurs ressources étant utilisées par un même étudiant, le pourcentage d'une ressource correspond à sa proportion d'utilisation pour l'année concernée.

Quatre ressources sont jugées peu utiles par l'ensemble des étudiants des trois années de formation: les anciennes leçons qu'ils ont réalisées pour d'autres stages, les cahiers des élèves de leur classe de stage, les autres étudiants et les connaissances c'est-à-dire les personnes de leur entourage. 
Au Bloc 1, ce sont les ressources cours de la Haute École et formateurs qui sont les plus utilisées. Cependant, lorsqu'on observe l'évolution de l'utilisation de ces ressources sur le parcours de formation, on constate qu'elles sont de moins en moins exploitées. Ainsi, la ressource cours de la Haute École classée première au Bloc 1, passe de la deuxième place (B2) à la quatrième place (B3) quant à la ressource formateurs, elle évolue de la deuxième (B1) à la quatrième place (B2) pour n'être quasiment plus sollicitée au Bloc 3 ( 8 e position).

Un autre constat intéressant est l'importance qu'acquiert progressivement la ressource Internet. Au Bloc 1, elle est classée cinquième et stagne à la sixième place au Bloc 2 tandis qu'elle se hisse à la deuxième place au Bloc 3 .

Alors qu'au Bloc 1 les ressources prioritairement utilisées par les étudiants sont celles issues directement de la formation (cours de la Haute École et formateurs), nous observons chez les étudiants finissants un attrait pour des ressources périphériques, indépendantes du cursus. La ressource bibliothèque est celle que les étudiants du Bloc 3 privilégient au moment de la préparation de leur stage, suivie de la ressource Internet.

\section{Les objets recherchés par les étudiants dans les trois ressources qu'ils privilégient}

Les étudiants du Bloc 1 recherchent avant tout de la méthodologie (dans les cours de la Haute École: $12.8 \%^{3}$ ) et des idées (auprès des formateurs: $11.7 \%$ ). Leur préoccupation est également de trouver des sources valides dans livres de la bibliothèque (10.5\%). On perçoit chez eux une volonté de «bien faire» et d'appliquer ce qui a été appris au sein des instituts de formation:

Les Ateliers de formation professionnelle nous donnent des mises en situation ou autres (B1AVC43) ${ }^{4}$. Les formateurs nous donnent des conseils, des astuces pour réussir au mieux (B1AVF43). Les livres de la bibliothèque, ce sont des sources sures (B1AVB5).

Au Bloc 2, les étudiants sont toujours à la recherche de méthodologie (11,9\%) et d'idées (11.9\%) ainsi que d'exercices (11.9\%) qu'ils vont à présent surtout rechercher en bibliothèque. La validité des sources (8.5\%) reste une de leurs préoccupations mais cette fois c'est principalement auprès du maitre de stage qu'ils espèrent la trouver. Au-delà de la volonté de «bien faire» on observe, au Bloc 2, un intérêt pour les contenus à enseigner et leur maitrise à travers un objet de recherche qui se fait plus présent dans les données: la théorie et la matière à connaitre (10.1\%) qu'ils vont d'abord rechercher dans leurs cours:

Les livres comprennent les pistes didactiques (B2AVB20). C'est au maitre de stage que doivent plaire mes leçons (B2AVM6). Les cours apportent la matière indispensable à connaitre (B2AVC17).

C'est encore la recherche d'idées (13.3\%) et de sources fiables (13.3\%) qui anime les étudiants du Bloc 3 et qu'ils pensent trouver à la bibliothèque. Ils sont également en demande de conseils pour s'organiser (11.1\%) qu'ils vont chercher 
auprès de leur maitre de stage. Mais ce qui caractérise principalement ce groupe, c'est l'utilisation d'Internet pour y trouver des informations (6.6\%) facilement accessibles (6.6\%):

Je vais à la bibliothèque pour avoir de nouvelles idées (B3AVB30). Le maitre de stage, c'est le «modèle» à suivre pour réaliser notre stage (B3AVM26). Internet, ça regorge d'infos faciles d'accès (B3AVI17).

\section{Quelles sont les attentes de L'étudiant lorsqu'il mobilise la ressource formateur (personne-ressource et cours)?}

Pour répondre à cette question, nous observons à la fois les objets recherchés et les postures sollicitées par les étudiants quand ils sollicitent le formateur.

\section{Les attentes des étudiants lorsqu'ils utilisent la ressource formateur}

Les étudiants qui consultent leurs cours ou qui interpellent le formateur sont à la fois en attente de son aide et de son expertise. Ils recherchent de l'aide auprès de la personne qu'ils sollicitent principalement pour trouver des idées ou une manière de s'organiser. Ils recherchent une expertise en feuilletant leurs cours, espérant surtout y trouver un apport théorique ou une méthodologie appropriée au niveau des élèves qu'ils auront en stage:

Les formateurs nous guident vers là où il faut aller (B1AVF9). Ils sont là pour nous orienter vers le bon chemin (B3AVM12). Ils nous donnent des idées originales (B2AVM16). Tout le déroulement de la réalisation de leçon est dans les cours (B1AVC42). Les cours apportent la matière indispensable à connaitre (B2AVC17).

Leur perception de cette ressource change peu et est assez semblable pour les trois années de formation. Cependant, une nuance est observée pour les étudiants du Bloc 1 qui semblent rechercher une approbation du formateur. Se détachant peu de leur contexte scolaire d'apprentissage habituel, ils le sollicitent parce qu'il est la personne de référence:

Je vais voir les formateurs car ce sont eux qui nous forment (B1AVF38). C'est eux qui nous ont enseigné la matière concernée (B1AVF39).

\section{Les postures du formateur recherchées}

À l'analyse des données récoltées, nous constatons que les postures du formateur sollicitées par les étudiants sont fort semblables pour les trois années de formation.

Ce que nous observons d'emblée c'est la recherche constante, tout au long de la formation, des postures «conseiller» et "prendre en main»; le conseil étant prioritairement attendu. Nous constatons également une tendance vers l'autonomie qui parait se marquer en fin de formation par la sollicitation, même si elle est encore minime, de la posture «collaborer» et l'abandon de la posture «imposer» présente faiblement la première année. La posture «se positionner en second» n'apparait, quant à elle, dans aucun verbatim. 
Ajoutons que les étudiants ne sollicitent pas la posture «soutenir, réconforter, rassurer», ce qui ne veut pas dire qu'ils n'ont pas besoin d'être soutenus.

\section{Discussion}

En nous basant sur l'analyse des résultats, deux éléments de discussion sont proposés: l'un centré sur l'évolution de l'étudiant dans son parcours de formation, observée au travers des ressources mobilisées; l'autre axé sur la ressource formateur et son apport dans la réactivation des savoirs enseignés. C'est, rappelons-le, le point de vue de l'étudiant qui est ici pris en compte et qui donne aux résultats leur originalité.

\section{La formation initiale, un processus d'autonomisation en trois phases}

Les ressources que les étudiants disent utiliser au moment de la préparation de stage semblent confirmer leur progression vers l'autonomie à travers trois phases développementales (Bernal Gonzalez et al., 2018).

Ainsi, au Bloc 1, nous observons des étudiants qui se conforment aux apprentissages scolaires et veulent respecter et «bien faire» ce qu'on leur a enseigné. En utilisant principalement des ressources académiques (cours et formateurs), ils semblent vouloir reproduire des stratégies qui fonctionnaient dans leur scolarité antérieure (Beaupère, Boudesseul, \& Macaire, 2009). Leur identité enseignante est en éclosion et on les perçoit surtout guidés par la formation et les savoirs - les ressources incorporées, selon Le Boterf (2003) - qui en sont issus.

Arrivés au Bloc 2, ils semblent vivre une phase d'insécurité telle que décrite dans le modèle (Bernal Gonzalez et al., 2018) en recherchant principalement le «comment faire» c'est-à-dire les savoirs procéduraux (Gervais \& Leroux, 2011; Le Boterf, 2003). Face au premier long stage qu'ils ont à préparer, ils sont surtout préoccupés par les contenus à maitriser et les méthodologies à appliquer. Par contre, contrairement aux hypothèses explicatives de cette phase d'insécurité (Bernal Gonzalez et al., 2018), la gestion de la classe ne semble pas être la première inquiétude des étudiants que nous avons interrogés. En tout cas, leurs recherches au moment de préparer les activités de classe ne vont pas dans ce sens et ciblent des préoccupations orientées sur la didactique et la maitrise de la matière à enseigner. Ajoutons que les ressources académiques sont moins sollicitées tout en restant bien présentes dans leurs choix.

Enfin, au Bloc 3, les étudiants paraissent davantage autonomes, se montrant plus affirmés en dirigeant eux-mêmes leur formation par l'utilisation plus fréquente de ressources externes (Gervais \& Leroux, 2011; Le Boterf, 2003), préférées aux savoirs appris. Ainsi la bibliothèque et Internet deviennent leurs ressources privilégiées bénéficiant sans doute de leur accessibilité (Boubée, 2011). En effet, comme Malo (2005), nous pensons que les étudiants opèrent 
des choix stratégiques en ce moment intense de préparation. Ils semblent guidés par l'urgence vécue face aux stages qui s'enchainent et s'amplifient en cette dernière année de formation, si bien qu'on les voit utiliser les ressources opératoires à portée de main (Martin, 2004).

Sous l'angle non déficitaire adopté, nous observons l'étudiant en recherche d'équilibre tout au long des trois années de formation. Il prend des décisions, opère des choix afin de s'adapter au contexte dans lequel il se trouve et non prioritairement de correspondre aux normes établies par la formation, comme celle de la pertinence des ressources utilisées. À l'instar de Malo (2005, 2010), nous pensons que sa recherche de viabilité est fondée sur trois critères: l'efficacité ("ça marche»), le confort («je suis à l'aise») et la certitude («je sais justifier mes choix»). Si la conjugaison de ces trois critères produit l'équilibre, il semble qu'au Bloc 1, les étudiants soient davantage préoccupés par le critère de certitude («bien faire ce qu'on a appris»), au Bloc 2 le critère de confort parait primer ("être à l'aise avec la matière et la didactique») tandis qu'au Bloc 3 l'efficacité semble prendre le pas ("trouver rapidement des activités qui marchent»). En conséquence, on perçoit que chaque critère est plus particulièrement activé à une étape du cursus, faisant correspondre la recherche de viabilité aux phases développementales non linéaires du parcours de formation (Bernal Gonzalez et al., 2018) et aux déséquilibres spécifiques qui s’y vivent.

\section{Le formateur, un facilitateur des liens théorie-pratique}

Au moment de préparer son stage, l'étudiant semble animé par l'objet de sa recherche. Les ressources sollicitées pour le trouver fluctuent selon les années. Ainsi, par exemple, quand il souhaite trouver des sources valides, c'est principalement à la bibliothèque que l'étudiant du Bloc 1 se dirige; au Bloc 2 il pense surtout les trouver auprès du maitre de stage et revient à nouveau les chercher en bibliothèque une fois arrivé au Bloc 3. D'une année à l'autre, l'étudiant semble tâtonner entre les ressources ne parvenant sans doute pas encore à identifier l'apport de chacune dans sa recherche d'équilibre ou d'adéquation aux attentes de la formation. Néanmoins, une nuance peut être apportée à ce constat. Elle concerne la ressource maitre de stage qui est systématiquement associée à l'expérience et à la connaissance du terrain ainsi que la ressource formateur (personneressource et cours) sollicitée tout au long des trois années de formation pour répondre à un besoin d'aide ou d'expertise.

L'expertise recherchée auprès du formateur à travers les cours qu'il a donnés nous interpelle. En effet, ces résultats apportent une nuance aux constats de notre précédente étude (Deprit \& Van Nieuwenhoven, 2018) qui identifiaient ces ressources (formateurs et cours) comme celles délaissées par les étudiants finissants. Ici encore, nous observons que les étudiants de dernière année ne les utilisent pas dans leurs trois premiers choix mais, lorsqu'ils les exploitent, c'est notamment pour réactiver des savoirs appris (méthodologie et théorie), 
tout comme le font les étudiants des deux premières années de formation. Cette réactivation des savoirs s'observe également lorsqu'ils feuillètent leurs cours à la recherche de la matière à connaitre. Cependant, indépendamment de ces recherches, l'établissement des liens entre la théorie et la pratique ne semble pas faire partie de leurs préoccupations, en tout cas, ils ne l'expriment pas.

Dès lors, est-ce en activant la ressource formateur que les liens théoriepratique, empreints de réflexivité sur l'action (Piot, 2008), sont élaborés? Ou bien ces liens sont-ils éclipsés par l'urgence de la situation qui pousse les étudiants à tenter de trouver, dans leurs cours, des méthodologies toutes faites ainsi que la matière à enseigner déjà transposée pragmatiquement par les formateurs, la rendant, comme le souligne Perrenoud (2004), directement accessible aux élèves? Ces questionnements restent à approfondir pour comprendre ce qui se joue au moment où l'étudiant construit ses activités d'apprentissage et, de là, discerner l'utilité et la nature d'un accompagnement à mettre en place dès la phase de planification, pour soutenir le stagiaire dans sa réflexion.

Ce qui semble certain, c'est que les étudiants sont en attente d'aide afin d'obtenir rapidement des idées efficaces (méthodologie, organisation) et d'arriver à concevoir, en temps voulu, des situations d'apprentissage qui les satisfassent pour maintenir ou retrouver ce qui, pour eux, fait équilibre et rend la situation viable dans ce rythme soutenu d'avant-stage.

\section{Conclusion}

L'objectif de cette étude était de déterminer ce que recherchent les étudiants au moment de préparer leur stage, notamment lorsqu'ils activent la ressource formateur (en tant que personne-ressource ou par l'intermédiaire de ses cours). À travers leur perception, nous souhaitions comprendre ce qui fonde leurs prises de décision pour trouver des façons viables de s'adapter aux situations qu'ils rencontrent. Pour ce faire, nous avons, d'une part, observé ce que les étudiants des trois années de formation recherchent dans les ressources priorisées au moment de la préparation de leur stage et, d'autre part, déterminé les raisons qui poussent les étudiants à utiliser la ressource formateur.

Comme nous le rappelions au début de cet article, peu d'écrits illustrent la phase préactive d'un stage (Clerc \& Martin, 2011). Sans avoir l'ambition de généraliser, l'apport de cette étude est double.

Premièrement, quand on regarde l'exploitation des ressources au moment de la préparation de leçons, on perçoit une évolution dans la recherche d'équilibre (besoins de certitude en B1, de confort en B2 et d'efficacité en B3) qui pourrait être liée aux phases de développement pré-professionnel que l'étudiant parcourt tout au long de son cursus (Bernal Gonzalez et al., 2018). Associée également aux phases développementales, on perçoit une autonomisation qui semble s'installer au gré du parcours de formation. En effet, à part au Bloc 1, les étudiants n'ont 
pas le réflexe de se diriger en premier vers les ressources issues de leur formation (formateurs et cours) pour rechercher ce qui les préoccupe principalement: trouver des idées et des méthodologies. On les voit s'en détacher peu à peu au profit de ressources qu'ils peuvent gérer seuls (bibliothèque et Internet). Cette émancipation professionnelle est par ailleurs observée à travers une tendance à solliciter des postures (Mieusset, 2013) de moins en moins directives, davantage collaboratives lorsqu'ils activent le réseau d'expertise.

L'utilisation d'Internet questionne quand on la voit culminer en tête des ressources utilisées par les étudiants comme un aboutissement final de leur formation. Ce phénomène pourrait être lié aux habitudes de vie des digital natives dont le quotidien est connecté et numérisé (Pedró, 2012) ou révéler une recherche active, encore tâtonnante, d'activités validées par l'expérience (Vause, 2011) les amenant à construire des situations d'apprentissage "puzzle» à partir de parties de synthèses trouvées ça et là, de manière organisée ou hasardeuse, dans des manuels ou sur la toile (Coppé, 2007). Pour cette raison, il est important de considérer les prises de décisions, en fonction de l'équilibre que chaque étudiant recherche et de sensibiliser les formateurs à cette approche afin qu'ils puissent comprendre la manière dont les étudiants fonctionnent et adapter leurs interventions aux réels besoins des futurs enseignants. Dans ce sens, distinguer les enjeux des étudiants dans l'exploitation des ressources en observant la manière dont ils mettent en balance ce qui les satisfait (principe de viabilité) de ce qu'il convient de faire (normes) est une piste de recherche à explorer.

Deuxièmement, la place de la ressource formateur en tant qu'aide à la réflexion pour réinvestir la théorie dans la pratique est questionnable au moment de la planification. Ainsi, nous constatons que cette ressource, d'office associée à une attente d'aide et d'expertise et ce, quelle que soit l'année de formation, n'est pas pour autant privilégiée. Si son exploitation est effectivement utilisée pour accéder aux savoirs académiques (méthodologie et théorie), nos analyses ne permettent pas de garantir que c'est au moment de leur sollicitation que la réflexion sur l'action se réalise et que les liens théorie-pratique attendus se construisent (Caron \& Portelance, 2017), ni affirmer que les étudiants y recherchent des théories formalisées. Il est probable, en effet, que les stagiaires espèrent y trouver des savoirs, certes, mais qui ont déjà fait l'objet d'une transposition pragmatique (Perrenoud, 2004), ainsi que des méthodologies directement utilisables favorisant un gain de temps (Martin, 2004). Dans cette optique, la nécessité d'investiguer plus largement l'équilibre entre les coûts et les bénéfices perçus par l'étudiant lorsqu'il utilise une ressource devient une perspective de recherche intéressante pour comprendre ses choix et approfondir nos connaissances sur la construction de la compétence de planification.

En conclusion, il parait indispensable d'être attentifs à la dynamique interne de l'étudiant pour proposer un soutien à l'exploitation pertinente des ressources, et de questionner la formation sur la place effective qu'elle accorde à l'articulation théorie-pratique. Ces liens devraient idéalement être activés dès la prépa- 
ration du stage. Mais doivent-ils être laissés à l'entière responsabilité de l'étudiant dès sa première expérience professionnelle? De plus, qui l'accompagne? Est-ce le rôle privilégié du formateur de l'institut de formation ou bien faut-il penser un accompagnement organisé en réseau (formateur et maitre de stage), tel que pensé par Colognesi et al. (2018), valorisant l'expertise de chacun à travers la complémentarité des gestes partagés (Colognesi \& Van Nieuwenhoven, 2017, sous presse)? Ce co-accompagnement au moment de la planification pourrait être une piste qui répondrait aux besoins des étudiants tiraillés entre les deux milieux de formations qui fonctionnent parfois en vases clos.

En terme de limites, notons que cette recherche constitue un premier traitement des données qu'il conviendra d'approfondir par la suite; le choix d'un questionnaire pour leur récolte nous fournit également peu de précisions sur les objets recherchés. Nous souhaitons donc, d'une part, poursuivre cette étude de manière compréhensive en choisissant des outils de recueil qui permettent de dépasser les discours des étudiants et donnent accès à leur activité réelle de planification (notamment au moyen de l'outil photovoice). D'autre part, nous souhaitons compléter notre précédente recherche (Deprit \& Van Nieuwenhoven, 2018) afin de déterminer, sur un échantillon plus important et dans une approche ergonomique de l'activité, ce qui constitue l'équilibre des étudiants au moment de développer leur compétence de planification.

\section{Notes}

1 Dans cet article, le terme formateur désigne l'enseignant de l'institut de formation, expert d'une discipline d'enseignement (mathématiques, éveil historique, pédagogie...).

2 En Belgique francophone, depuis le décret Paysage (2013), les trois années de formation sont appelées Blocs: Bloc 1 (B1), Bloc 2 (B2), Bloc 3 (B3).

3 Les pourcentages correspondent à la proportion d'utilisation de la ressource pour l'objet recherché.

4 Codage du verbatim.

\section{Références}

ARES. (2017). Avis de l'ARES n²017-17: avant projet de décret définissant la formation initiale des enseignants (2017). Repéré à http://www.ares-ac.be/fr/a-propos/instances/ conseil-d-administration/avis\#2017

Beaupère, N., Boudesseul, G., \& Macaire, S. (2009). Sortir sans diplôme de l'université. De l'orientation post-bac à l'entrée sur le marché du travail. Observatoire national de la vie étudiante. Infos, 21,1-8. Repéré à http://www.ove-national.education.fr/medias/files/ ove-infos/oi21_ove_infos_21-_bat_final_page_7-4-9.pdf

Bernal Gonzalez, A., Houssa Cornet, M.-C., Kinet, A., Labalue, F., Salamon, A.-J., Zuanon, E., \& Deprit, A. (2018). Les difficultés pressenties par les futurs instituteurs en cours de formation initiale. In F. Dufour, L. Portelance, C. Van Nieuwenhoven \& I. Vivegnis (Éds.), La préparation à l'insertion professionnelle en enseignement. Montréal, Québéc: Presses de l'Université du Québec.

Boubée, N. (2011, octobre). Caractériser les pratiques informationnelles des jeunes: Les problèmes laissés ouverts par les deux conceptions «natifs» et «naïfs» numériques. Document présenté dans les 5e Rencontres Savoirs CDI à Rennes. Resumé consulté dans https:// 
www.reseau-canope.fr/savoirscdi/fileadmin/fichiers_auteurs/Actes/Rennes_2011/NBRencontresSavoirsCDI-oct2011.pdf

Caron, J., \& Portleance, L. (2017). Perceptions de stagiaires quant au soutien des deux formateurs à l'articulation entre théorie et pratique. Formation et profession, 25(1), 34-49. doi:10.18162:fp.2017.367

Clerc, A., \& Martin, D. (2011). L'étude collective d'une leçon, une démarche de formation pour développer et évaluer la construction des compétences professionnelles des futurs enseignants. Revue internationale de pédagogie de l'enseignement supérieur, 27(2), 1-16. Repéré à http://ripes.revues.org/514

Colognesi, S., Beausaert, S., \& Van Nieuwenhoven, C. (2018). L'accompagnement des enseignants tout au long de la carrière défini autour de quatre fondements. In C. Van Nieuwenhoven, S. Colognesi, \& S. Beausaert (Éds.), Accompagner les pratiques des enseignants. Un défi pour le développement professionnel en formation initiale, en insertion et en cours de carrière (pp. 5-14). Louvain-la-Neuve, Belgique: Presses universitaires de Louvain.

Colognesi, S., \& Van Nieuwenhoven, C. (sous presse). Un groupe de recherche collaborative comme levier de développement des compétences professionnelles et de (re)connaissance du méter. In P. Guibert, X. Dejemeppe, J. Desjardins, O. Maulini (Éds.), La formation des enseignants, amie critique de la profession? (pp. 84-94). Bruxelles, Belgique: De Boeck.

Coppé, S. (2007). La préparation des séances en classe par les stagiaires en fin de formation: un exemple de résistances et de changements dans les pratiques. In La statistique dans l'enseignement PLC2 \& Les résistances et les changements dans les pratiques d'enseignement en formation initiale: actes du $14^{e}$ colloque de la CORFEM (pp. 165-181). Versailles, France. Repéré à http://www.univ-irem.fr/exemple/corfem/Actes_2007_11.pdf

Correa Molina, E., Gervais, C., \& Rittershaussen, S. (2008). La recherche sur la formtion en milieu de pratique: occaion d'un regard critique. In E. Correa Molina, C. Gervais, \& S. Rittershaussen (Éds.), Vers une conceptualisation de la situation de stage: explorations internationales (pp. 11-19). Sherbrooke, Québec: Éditions du CRP.

Décret 2000: décret de la Communauté française du 12 décembre 2000 définissant la formation initiale des instituteurs et des régents. (2000). Moniteur belge, 19 janvier 2001, Lois 25501.

Décret Paysage: décret de la Communauté française du 7 novembre 2013 définissant le paysage de l'enseignement supérieur et l'organisation académique des études. (2013). Moniteur belge, le 18 décembre 2013, Docu nº 39681.

De Ketele, J.-M. (2018). Former et accompagner les pratiques professionnelles des enseignants en formation initiale, en insertion et en cours de carrière. In C. Van Nieuwenhoven, S. Colognesi, \& S. Beausaert (Éds.), Accompagner les pratiques des enseignants. Un défi pour le développement professionnel en formation initiale, en insertion et en cours de carrière (pp. 15-25). Louvain-la-Neuve, Belgique: Presses universitaires de Louvain.

Deprit, A., \& Van Nieuwenhoven, C. (2018). Comment les étudiants se préparent-ils aux stages. Ressources exploitées et choix stratégiques, Revue canadienne de l'éducation, 41(3), 726-752.

Dessus, P. (2002). Description et prescription dans les méthodes de recherche en éducation. In J.-F. Marcel (Éd.), Les sciences de l'éducation, des recherches, une discipline (pp. 113-130). Paris, France: L'Harmattan.

Dweck, C. S. (2003). Ability conceptions, motivation and development. British Journal of Educational Psychology [Special Issues: Motivation and Development], 13-27.

Fleming, J. (2014). Engaging beginning teachers as experts in professionnal development, The New Educator, 10(3), 166-181. doi: 10.1080/1547688x.2014.92

Gervais, C. (2008). Pour communiquer son expérience: l'explication argumentée des savoirs d'enseignants associés et des stagiaires. In E. Correa Molina, C. Gervais, \& S. Rittershaussen (Éds.), Vers une conceptualisation de la situation de stage: explorations internationales (pp. 191-204). Sherbrooke, Québec: Éditions du CRP. 
Gervais, C., \& Leroux, M. (2011). Ressources mobilisées par des stagiaires pour enseigner: faible part des savoirs théoriques. Revue des sciences de l'éducation, 37(2), 281-306. doi: $10.7202 / 1008987 \mathrm{ar}$

Le Boterf, G. (2003). Développer la compétence des professionnels. Construire des parcours professionnalisants. Paris, France: Éditions d'Organisation.

Malo, A. (2005). Parcours évolutif d'un savoir professionnel: une étude de cas multiples menée auprès de futurs enseignantes et enseignants du secondaire en stage intensif (thèse de doctorat). Université Laval, Québec, Canada.

Malo, A. (2010). Appréciation des stagiaires au sujet des apprentissages effectués en contexte de stage en enseignement, Éducation et francophonie, 38(2), 78-95.

Martin, D. (2004). Les terrains de la formation des maitres et de la réforme curriculaire: une perspective anthropologique sur le développement de l'expertise enseignante. In C. Lessard, M. Altet, L. Paquay, \& P. Perrenoud (Éds.), Entre sens commun et sciences humaines. Quels savoirs pour enseigner? (pp. ??-??). Bruxelles, Belgique: De Boeck.

Mieusset, C. (2013). Les dilemmes d'une pratique d'accompagnement et de conseil en formation. Analyse de l'activité réelle du maitre de stage dans l'enseignement secondaire (thèse de doctorat). Université de Reims Champagne-Ardenne, Reims, France.

Monfort, V. (2006). Repenser l'échec à l'université. Les Temps Modernes, 3(637-638-639), 703-715. doi:10.3917/ltm.637.0703

Paquay, L. (2012). Continuité et avancées dans la recherche sur la formation des enseignants. Les Cahiers de Recherche du Girsef, 90, 1-36. Repéré à https://hal.archives-ouvertes.fr/ hal-00978949

Paquelin, D. (2016). Attentes et pratiques des étudiants dans l'enseignement supérieur français. Note de synthèse. Récupéré de la plateforme pédagogique de l'Université Paris Descartes. Repéré à https://moodle-admin.parisdescartes.fr $/ \mathrm{mod} /$ resource $/$ view.php?id=11540

Paul, M. (2016). La démarche d'accompagnement. Repères méthodologiques et ressources théoriques. Louvain-la-Neuve, Belgique: De Boeck supérieur.

Pedró, F. (2012). Connected Minds. Technology and Today's Learners. Paris, France: OCDE.

Perrenoud, P. (2004). Les sciences de l'éducation proposent-elles des savoirs mobilisables? In C. Lessard, M. Altet, L. Paquay, \& P. Perrenoud (Éds.), Entre sens commun et sciences humaines. Quels savoirs pour enseigner? (pp.139-157). Bruxelles, Belgique: De Boeck.

Piot, T. (2008). La construction des compétences pour enseigner. Revue des sciences de l'éducation de McGill, 42(2), 95-110.

Ria, L. (2016). Lexique. In L. Ria (Éd.), Former les enseignants au XXIe siècle. Professionnaité des enseignants et de leurs formateurs (pp. 249-257). Louvain-la-Neuve, Belgique: De Boeck Supérieur.

Saujat, F., Saussez, F., \& Sève, C. (2016). Activités des formateurs d'enseignants: quelles fonctions pour quels objectifs? Éléments de synthèse. In L. Ria (Éd.), Former les enseignants au XXIe siècle. Professionnaité des enseignants et de leurs formateurs (pp. 235-242). Louvainla-Neuve, Belgique: De Boeck Supérieur.

Savoie-Zajc, L. (2011). La recherche qualitative/interprétative en éducation. In T. Karsenti \& L. Savoie-Zajc (Éds.), La recherche en éducation; étapes et approches (pp. 123-147). St-Laurent, Québec: ERPI.

Savolainen, R. (1999). The role of Internet in information seeking. Putting the networked services in context. Information Processing and Management, 35(6), 765-782.

Schön, D.A. (1983). The reflexive practitioner. How professionals think in action. New-York, NY: Basic Book.

Ulvik, M., \& Langørgen, K. (2012). What can experienced teachers learn from newcomers? Newly qualified teachers as a resource in schools, Teachers and Teaching, 18(1), 43-57. doi:10.1080/13540602.2011.622553

Van der Maren, J.-M. (1996). Méthodes de recherche pour l'éducation (2e éd.). Montréal, Québec: PUM. 
Van Nieuwenhoven, C., Picron, V., \& Colognesi, S. (2016). Accompagner les premiers pas des stagiaires de terrain: quels enjeux et quelles tensions pour les formateurs? In L. Ria (Éd.), Former les enseignants au XXIe siècle. Volume 2: Professionnalité des enseignants et de leurs formateurs (pp. 139-150). Bruxelles, Belgique: De Boeck Supérieur.

Vause, A. (2011). Des pratiques aux connaissances pédagogiques des enseignants: les sources et les modes de construction de la connaissance ouvragée (thèse de doctorat). Université catholique de Louvain, Louvain-la-Neuve, Belgique.

Vial, M., \& Caprarros-Mencacci, N. (2007). L'accompagnement professionnel? Méthode à l'usage des praticiens exerçant une fonction éducative. Bruxelles, Belgique: De Boeck.

Waege, K., \& Haugaløkken, O. K. (2013). Research-based and hands-on practical teacher education. An attempt to combine the two. Journal of Education for Teaching, 39(2), 235-249.

Mots-clés: Préparation de stage, articulation théorie-pratique, formateurs, postures, ressources utilisées

\section{Welche Rolle spielen Ausbilder für Studierende bei der Praktikumsvorbereitung?}

\section{Zusammenfassung}

Eine Verknüpfung zwischen Theorie und Praxis während der Praktikumsvorbereitung herzustellen fällt Studiernden of schwer. Häufig ist ein grosser Unterschied zwischen dem erlernten Wissen und der Realität der Umsetzung auf dem Gebiet zu beobachten (Caron \& Portelance, 2017). Um zu untersuchen, ob Ausbilder diese Verknüpfungen fördern können, wurde mittels eines Fragebogens erfasst, welche Ressourcen und Mittel Studierende bei der Praktikumsvorbereitung bevorzugen, Die Untersuchung zeigt, dass sich Studierende gegen Kursende, nach und nach vom Ausbilder und den Unterrichten lösen, um vorwiegend externe Ressourcen (Bibliothek und Internet) heranzuziehen.

Schlagworte: Praktikumsvorbereitung, Artikulierung Theorie-Praxis, Ausbilder, genutzte Ressourcen 


\section{Nell'ambito delle risorse a disposizione di uno studente per pianificare il suo tirocinio, qual'è il ruolo riservato al tutor didattico?}

Riassunto

L'articolazione tra teoria e pratica durante la preparazione di un tirocinio è difficile per lo studente poiché egli percepisce un divario tra le conoscenze apprese e la realtà sul terreno (Caron \& Portelance, 2017). Per esaminare se il formatore rappresenta la risorsa attraverso la quale lo studente concretizza tali collegamenti, una ricerca esplorativa ha permesso, tramite un questionario scritto, di identificare le risorse prioritarie che lo studente utilizza per preparare il suo tirocinio. L'analisi evidenzia che lo studente si distacca gradualmente dal formatore e dai suoi corsi e, verso la fine del percorso formativo, tende a utilizzare maggiormente risorse esterne alla formazione (biblioteca e Internet).

Parole-chiave: Preparazione del tirocinio, articolazione tra teoria e pratica, formatori, risorse utilizzate

\section{Amongst the Resources Mobilised By The Student To Plan His Traineeship, What Role Does He Foresee for His Institutional Trainer?}

\section{Summary}

Theoretical-practical articulation at the time of the preparation of an internship is difficult for students as there is a perceived gap between academic knowledge and the reality in the field (Caron \& Portelance, 2017). In order to determine whether the trainer is the resource through which students bridge this gap, exploratory research was conducted by means of a written questionnaire. The questionnaire facilitated the identification of the resources prioritized by students who are preparing for internships. The analysis shows that students gradually distance themselves from the trainer and their coursework until they use, at the end of the curriculum, resources more external to training (library and Internet).

Keywords: Internship preparation, theory-practice articulation, trainers, postures, used resources 
Agnès Deprit, étudiante au doctorat, chercheure au GIRSEF (Groupe Interdisciplinaire de Recherche sur la Socialisation, l'Éducation et la Formation). Domaine de recherche: la formation des enseignants.

Université catholique de Louvain (UCLouvain), Grand Place 43, B-Louvain-laNeuve.

E-Mail: agnes.deprit@uclouvain.be

Virginie März, docteure en sciences de l'éducation, professeure à la faculté de psychologie et des sciences de l'éducation, chercheure au GIRSEF. Domaine de recherche: les curriculums et le développement professionnel.

Université catholique de Louvain (UCLouvain), Grand Place 43, B-Louvain-laNeuve.

E-Mail: virginie.marz@uclouvain.be

Catherine Van Nieuwenhoven est docteur en sciences de l'éducation et professeur à l'UCLouvain. Ses recherches portent sur l'accompagnement des pratiques professionnelles, sur l'insertion professionnelle des enseignants et sur l'évaluation.

Université catholique de Louvain (UCLouvain), Grand Place 43, B-Louvain-laNeuve.

E-Mail: catherine.vannieuwenhoven@uclouvain.be 